\title{
Boundary Finding with Curve Embedding Potential Field
}

\author{
Gary H.P. Ho and Pengcheng Shi \\ Biomedical Research Laboratory \\ Department of Electrical and Electronic Engineering \\ Hong Kong University of Science and Technology \\ Clear Water Bay, Kowloon, Hong Kong \\ \{garyho, eeship\}@ust.hk
}

\begin{abstract}
We introduce an implicit vector field representation for arbitrary number of closed curves in space. Each curve-defining vector of the Curve Embedding Potential Field (CEPF) is defined to be perpendicular to the nearest curve, with its magnitude determined by its distance to that curve. Thereafter, we present an image segmentation strategy through the detection of the CEPF distortion caused by vectorform image data constraints. The CEPF-based method allows grid-free localization of curve elements at any detection stage, while maintaining the advantages of being geometric in nature. Unlike the level set methods, the evolution of the embedded curves is not constrained in any particular directions, and the implementation is straightforward. We discuss the accuracy and robustness of the algorithms under different noise conditions, and present segmentation results of various medical images, including MRI, ultrasound, and mammogram. ${ }^{1}$
\end{abstract}

\section{Introduction}

Earlier efforts of deformable and geodesic active contours set default directions for curve evolution [2,4]. For example, the Snakes would keep shrinking inwards in order to minimize the internal energy term in its formulation, and the level set active contour [5] always expands or contracts by moving individual front points in their normal directions, while the image edginess information is treated as external data constraint that distorts normal ways of curve evolution. This type of formulation increases the dependency on the initial contour position, and some regions in an image may never be reached if the initialization is not appropriate.

Enhancements have been made to make the evolving curve less sensitive to its initial position, such as the introduction and adoption of the gradient vector flow (GVF) as data constraints $[6,8]$. The GVF is computed as a diffusion of image gradient vectors and it increases the domain of influence for imagederived edge information. Every pixel $\mathbf{p}$ of the image is affected by all edge

\footnotetext{
${ }^{1}$ For better viewing quality, the color version of the figures can be found at http://eesg2.ee.ust.hk/ gary/cepf_miccai_color.pdf
} 
points at different levels, where the influence of different edge points is inversely proportional to the Euclidean distance from $\mathbf{p}$ to the edge, i.e. closer edge point has stronger effect. The output of the GVF process is a smooth vector field with each vector indicating the most likely direction towards an edge and the vector length denoting how close the edge is, longer means closer (see middle columns of Figure 6 for examples). Incorporation of the GVF as data constraints into the level set formulation has been attempted [6], and it has shown greater flexibility on initial contour positioning. The main advantage is that the evolving contour can move in the reversed normal direction by taking the inner product between the front normal $N$ and the GVF vector $v$ on the same location as the active contour evolving velocity $C_{t}=(v \cdot N) N$. However, additional constraints in the formulation are needed to handle the cases where the inner product $v$. $N$ is zero, which means that the GVF vector is tangent to the evolving front and therefore the front does not move smoothly while maintains its structural integrity. Although the effect of zero inner product can be reduced by using a more complicated level set formulation [6], the nature of the edge information (including the GVF) is still passive, static, and usually scalar external constraint on the evolving curve and its effective usage is controlled by the status of the current front. Typically, the vector form edge information such as the gradient and the GVF is first reduced to scalar form by taking inner product or using its magnitude, and hereafter applied to a contour dependent normal direction. It is definitely a reduction of source information and hence may limit the accuracy and suitability of the active contour models in many situations.

In the following presented method, we focus on utilizing the vector form edge information directly on object segmentation while simplifying the complicity of contour model evolution. The keys are to operate on native vector form data constraints and to alleviate unnecessary structure-maintaining constraints, which lead to simpler implementation. Inspired by the vector level set ideas in computational mechanics [7], we introduce an implicit vector field representation of active contours, the Curve Embedding Potential Field, which allows the merge and break of the curves and can handle discontinuities. Based on previous active contour models $[1,6]$, we use positional vectors which give the distances and normal directions to their nearest front to replace the level set function.

\section{Methodology}

The CEPF segmentation is an iterative process (see Fig. 1 for the flowchart of the framework), with the core idea being that active contours are implicitly represented by a potential field where locations with zero potential represent the embedded continuous curves. Starting from an initial closed curve placed roughly around the interested object(s) through prior knowledge of the boundary location or user interaction, the CEPF of this initial contour is created through a curveto-field transformation. Under the influence of the image-derived, vector-form edge information, the initial CEPF would be distorted and the underlying curve would deform and propagate towards the object boundaries. At the end of the 


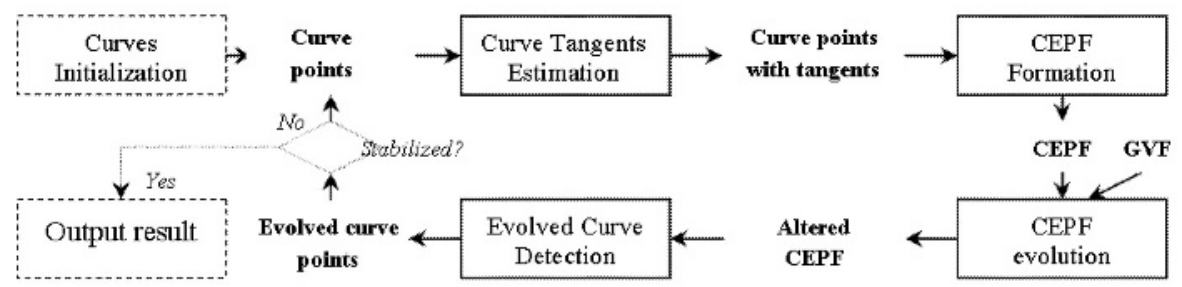

Fig. 1. Overall flowchart of the framework. Rectangular blocks are the major process steps and the boldface letters are the corresponding input and output between steps.

process, the stabilized CEPF and the corresponding curves would indicate the object boundaries.

Estimation of Curve Tangents: The curve-to-field transformation requires the tangents of each curve element. In other words, we need to determine the tangent directions of a set of discrete curve points which represent the continuous curve. In our implementation, the tangent of curve point $\mathbf{z}$ is estimated using Equation 2, which uses the relative positions of the two nearest neighboring curve points $\mathbf{z}_{1}$ and $\mathbf{z}_{2}$ to $\mathbf{z}$. Further, an end-point checking procedure is needed in order to maintain the smoothness of the CEPF field:

$$
\begin{aligned}
\operatorname{endPt}(\mathbf{z}) & = \begin{cases}1, & \text { if }\left(\mathbf{z}_{2}-\mathbf{z}_{1}\right) \cdot\left(\mathbf{z}_{1}-\mathbf{z}\right)>0 \\
0, & \text { otherwise }\end{cases} \\
\text { Tangent }(\mathbf{z}) & = \begin{cases}\left(\mathbf{z}_{1}-\mathbf{z}\right) /\left(\left|\mathbf{z}_{1}-\mathbf{z}\right|\right), & \text { if } \operatorname{endPt}(\mathbf{z})=1 \\
\left(\mathbf{z}_{2}-\mathbf{z}_{1}\right) /\left(\left|\mathbf{z}_{2}-\mathbf{z}_{1}\right|\right), & \text { otherwise }\end{cases}
\end{aligned}
$$

Formation of the Curve Embedding Potential Field: Construction of the CEPF on each grid/pixel point $\mathbf{p}$ on the image requires the two closest curve points $\mathbf{z}_{1}$ and $\mathbf{z}_{2}$ to $\mathbf{p}$, as illustrated in Figure 2 . Let $\mathbf{t}_{1}=\operatorname{Tangent}\left(\mathbf{z}_{1}\right)$ and $\mathbf{t}_{2}=$ Tangent $\left(\mathbf{z}_{2}\right)$ from Equation 2, we have

$$
\begin{aligned}
\mathbf{n}_{1} & =\left(\left(\mathbf{z}_{1}-\mathbf{p}\right) \cdot \mathbf{t}_{1}\right) \mathbf{t}_{1}+\left(\mathbf{z}_{1}-\mathbf{p}\right) \\
\mathbf{n}_{2} & =\left(\left(\mathbf{z}_{2}-\mathbf{p}\right) \cdot \mathbf{t}_{2}\right) \mathbf{t}_{2}+\left(\mathbf{z}_{2}-\mathbf{p}\right) \\
C \operatorname{EPF}(\mathbf{p}) & = \begin{cases}\mathbf{0}, & \text { if } \min \left(\operatorname{dist}\left(\mathbf{p}, \mathbf{z}_{1}\right), \operatorname{dist}\left(\mathbf{p}, \mathbf{z}_{2}\right)\right)=0 \\
\left(\mathbf{n}_{1}+\mathbf{n}_{2}\right) / 2, & \text { otherwise }\end{cases}
\end{aligned}
$$

Here, $\mathbf{n}_{1}$ is the vector starting from $\mathbf{p}$ and is orthogonal to the tangent of the closest curve point $\mathbf{z}_{1}, \mathbf{n}_{2}$ is the same type of vector for $\mathbf{z}_{2}$, dist() is the geometric distance measure, and $C E P F(\mathbf{p})$ takes the average of these two vectors in order to handle the corner cases.

CEPF Evolution and Curve Propagation: Once we have transformed the curve into a CEPF field, instead of directly moving the curve elements, we propagate the embedded curve by applying image-derived vector-form boundary information such as GVF on the CEPF. Such a distortion to the potential field 

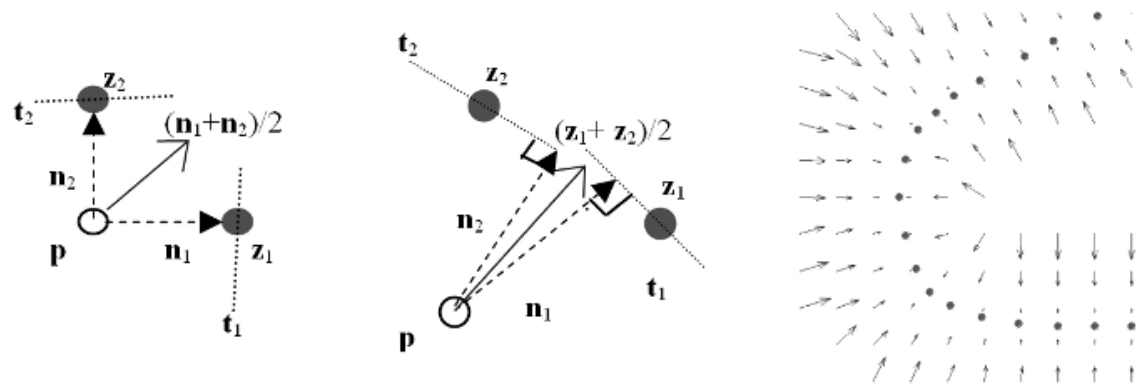

Fig. 2. Formation of the CEPF. White circles $\mathbf{p}$ represent arbitrary grid points on the image. Points $\mathbf{z}_{1}$ and $\mathbf{z}_{2}$ are the two nearest curve points to $\mathbf{p}$, and $\mathbf{t}_{1}$ and $\mathbf{t}_{2}$ are their associated tangents (dotted lines) respectively. Diagram on the left shows a CEPF vector (solid arrow) formed near curve corner, and another CEPF vector near smooth curve is shown in the middle. An example of the constructed CEPF within a narrow band is shown on the right, where the solid dots denote samples of the embedded curve.

changes the status of the embedded curve implicitly, and we can then extract the updated positions of those curve elements from the distorted CEPF.

Assuming that we have a smooth CEPF field $\mathbf{f}(\mathbf{x})$ where $\mathbf{x} \in \mathbb{R}^{2}$, the CEPF vectors point to the corresponding nearest curve positions orthogonally (see Figure 3). Given an image-driven edge field $\mathbf{g}(\mathbf{x})$ and the current CEPF field $\mathbf{f}^{t}(\mathbf{x})$, we estimate the updated CEPF field $\mathbf{f}^{t+1}(\mathbf{x})$ using the finite difference procedure through the following basic CEPF evolution equations for an arbitrary point $\mathbf{p}$ on a sampling grid:

$$
\begin{array}{ll}
\text { distort: } & \mathbf{d}^{t+1}(\mathbf{p})=\mathbf{f}^{t}(\mathbf{p})+\Delta t \mathbf{g}\left(\mathbf{p}+\mathbf{f}^{t}(\mathbf{p})\right) \\
\text { tangent: } & \mathbf{t}^{t+1}(\mathbf{p})=\left(\mathbf{p}+\mathbf{d}^{t+1}(\mathbf{p})\right)-\left(\mathbf{p}_{i}+\mathbf{d}^{t+1}\left(\mathbf{p}_{i}\right)\right) \\
\text { restore: } & \mathbf{f}^{t+1}(\mathbf{p})=\mathbf{d}^{t+1}(\mathbf{p})-\left(\mathbf{d}^{t+1}(\mathbf{p}) \cdot \mathbf{t}^{t+1}(\mathbf{p})\right) \frac{\mathbf{t}^{t+1}(\mathbf{p})}{\left|\mathbf{t}^{t+1}(\mathbf{p})\right|^{2}}
\end{array}
$$

Equation 6 is the physical movement of a curve element relative to $\mathbf{p}$. Since $\mathbf{g}$ is independent of current $\mathbf{f}^{t}$, it can be viewed as an external distortion to $\mathbf{f}^{t}$. However, it maybe not comply with the integrity of being a potential field, i.e. $\mathbf{d}^{t+1}(\mathbf{p}) \neq \mathbf{f}^{t+1}(\mathbf{p})$. In order to reconstruct $\mathbf{f}^{t+1}$ which requires the orthogonality to the embedded curves, neighboring curve elements have to be consulted in Equation 7. With proper selection of reference neighbor(s), the updated CEPF field can be obtained using Equation 8.

All vector values in the above three equations are well-defined using regular grid sampling. The remaining problem is the choice of reference neighbor $\mathbf{p}_{i}$ which is obviously related to the current status of the evolving $\mathbf{f}$ and the sampling frequency. In Figure 3 (middle), the direction of a potential vector on a particular grid point can be very different from the point next to it, and this situation fails to give a reasonable tangent for Equation 7. To alleviate the effect of inappropriate reference selection, we have used the average of the tangents resulting from all possible neighboring points on the sampling grid. Let $\mathbf{p}_{i}$, where 
$i=1,2,3,4$, be the immediate up, down, left and right neighbors of $\mathbf{p}$. The average tangent $\overline{\mathbf{t}}$ is then computed from:

$$
\begin{aligned}
\overline{\mathbf{t}}^{t+1}(\mathbf{p}) & =\frac{1}{4} \sum_{i=1}^{4}\left[\left(\mathbf{p}+\mathbf{d}^{t+1}(\mathbf{p})\right)-\left(\mathbf{p}_{i}+\mathbf{d}^{t+1}\left(\mathbf{p}_{i}\right)\right)\right] \\
& =\mathbf{p}-\frac{1}{4} \sum_{i=1}^{4} \mathbf{p}_{i}+\mathbf{f}^{t}(\mathbf{p})-\frac{1}{4} \sum_{i=1}^{4} \mathbf{f}^{t}\left(\mathbf{p}_{i}\right)+\Delta t\left[\mathbf{g}\left(\mathbf{p}+\mathbf{f}^{t}(\mathbf{p})\right)-\frac{1}{4} \sum_{i=1}^{4} \mathbf{g}\left(\mathbf{p}_{i}+\mathbf{f}^{t}\left(\mathbf{p}_{i}\right)\right)\right]
\end{aligned}
$$

Consider the general smoothness assumption on the CEPF field, which implies that the local average $\mathbf{f}\left(\mathbf{p}_{i}\right) \approx \mathbf{f}(\mathbf{p})$, the above expression is simplified to

$$
\overline{\mathbf{t}}^{t+1}(\mathbf{p}) \approx \Delta t\left[\mathbf{g}\left(\mathbf{p}+\mathbf{f}^{t}(\mathbf{p})\right)-\frac{1}{4} \sum_{i=1}^{4} \mathbf{g}\left(\mathbf{p}_{i}+\mathbf{f}^{t}\left(\mathbf{p}_{i}\right)\right]\right.
$$

Adopting an even stronger assumption that $\mathbf{f}^{t}\left(\mathbf{p}_{i}\right) \approx \mathbf{f}^{t}(\mathbf{p})$, we have

$$
\overline{\mathbf{t}}^{t+1}(\mathbf{p}) \approx-\Delta t\left[\operatorname{Laplacian}\left(\mathbf{g}\left(\mathbf{p}+\mathbf{f}^{t}(\mathbf{p})\right)\right)\right]
$$

Equation 10 implies a prediction of the tangent direction purely based on the data vector field, which might be less accurate than Equation 9. However, it offers substantial implementation advantages since it is independent of the evolving curve, and we only need to compute the Laplacian of the $\mathbf{g}$ field once.

To sum up, for each grid point $\mathbf{p}$, its CEPF evolves through

$$
\begin{aligned}
\mathbf{d}^{t+1}(\mathbf{p}) & =C E P F^{t}(\mathbf{p})+\Delta t \mathbf{g}\left(\mathbf{p}+C E P F^{t}(\mathbf{p})\right) \\
C E P F^{t+1}(\mathbf{p}) & =\mathbf{d}^{t+1}(\mathbf{p})-\left(\mathbf{d}^{t+1}(\mathbf{p}) \cdot \widehat{\mathbf{t}}^{t+1}(\mathbf{p})\right) \widehat{\mathbf{t}}^{t+1}(\mathbf{p})
\end{aligned}
$$

where $\Delta t$ is the time step that governs the speed of the evolution and $\mathbf{g}$ is the GVF data field. $\widehat{\mathbf{t}}$ is the normalized $\overline{\mathbf{t}}$ from either Equation 9 or 10 .

In practice, GVF divergence may cause curve elements to be separated apart and create end points, which means that the curve is no longer closed. Hence, as in Equation 1, the tangents of the end points are specially handled to ensure that the CEPF extends naturally beyond the end points. Newly detected curve points beyond the original end points would fill up the gaps between diverted points after CEPF re-formation. More curve points imply that the length of the embedded curves has increased. On the other hand, converging GVF would reduce the curve length in a similar fashion. Since we need to maintain the smoothness of $\mathbf{f}$, handle of the GVF convergence/divergence, and filter out noise, we iteratively move and reconstruct the CEPF until it is relatively stabilized.

Detection of Implicitly Embedded Curves: Once the distorted CEPF is computed, the evolved curve points are extracted near their locations at last iteration. The local averages of the evolved CEPF vectors are used as the detection metrics for the evolved curves:

$$
\begin{aligned}
\overline{\mathbf{p}}_{x, y} & =\sum_{i=x-1}^{x+1} \sum_{j=y-1}^{y+1} \frac{C E P F^{t+1}\left(\mathbf{p}_{i, j}\right)+\mathbf{p}_{i, j}}{9} \\
Z_{t+1} & =\left\{\overline{\mathbf{p}}_{x, y} \mid\left(\left|\overline{\mathbf{p}}_{x, y}-\mathbf{p}_{x, y}\right|\right)<0.5 \forall \mathbf{p}_{x, y} \in \mathbb{I}\right\}
\end{aligned}
$$



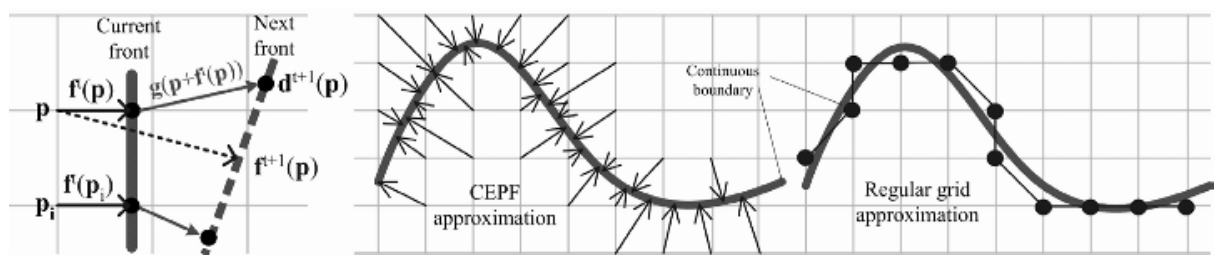

Fig. 3. Left: Basic model of CEPF evolution. Right: Comparison of curve representation between $\mathrm{CEPF}$ and regular grid sampling.

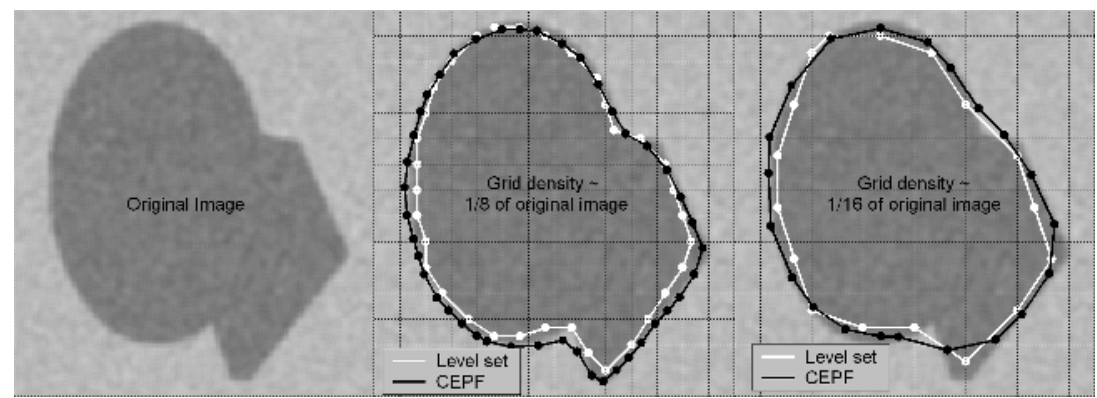

Fig. 4. Experimental illustration of difference in sampling between Levelset and CEPF.

Equation 13 gives a precise definition for the local averaging operation, and Equation 14 defines an acceptable region for new curve points around each grid point $\mathbf{p}_{x, y}$. After the detection of the evolved implicit curves, a new CEPF field is reconstructed from these new curve points for further processes until convergence (see Figure 1).

\section{Results and Discussions}

Comparison to Levelset Schemes: If we expand Equation 8 by substituting in Equation 6 and 10, we have:

$$
\mathbf{f}^{t+1} \approx \mathbf{f}^{t}+\Delta t\left[\mathbf{g}-\left(\left(\mathbf{f}^{t}+\Delta t \mathbf{g}\right) \cdot \nabla^{2} \mathbf{g}\right) \nabla^{2} \mathbf{g}\right]
$$

Compared to the basic Levelset formulation:

$$
\Psi_{t+1} \approx \Psi_{t}+\Delta t F\left|\nabla \Psi_{t}\right|
$$

where $\Psi$ is the distance function, $\nabla \Psi$ is the normal direction of the hypersurface from $\Psi$, and $F$ is a speed function in terms of image gradient and current status of $\Psi$ (e.g. curvature), the similarities are:

\begin{tabular}{c|cc} 
& Levelset & CEPF \\
\hline Embedding structure & $\Psi$ & $\mathbf{f}$ \\
Curve Elements & $\{\Psi=0\}$ & $\{|\mathbf{f}|=0\}$ \\
Speed & $F(\Psi,|G(I)|)$ & $\mathbf{g}$
\end{tabular}




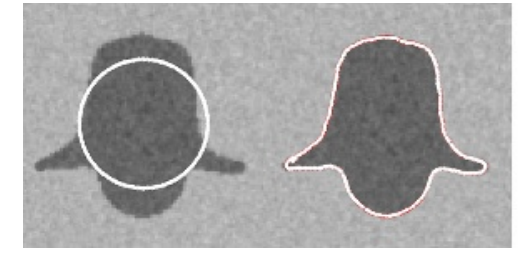

SNR $\mid$ Mean Error Standard Min Error Max Error

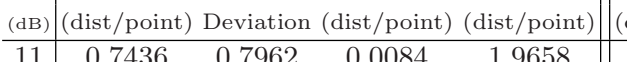

\begin{tabular}{l|lll}
9 & 0.6154 & 1.0339 & 0.0118
\end{tabular}

$\begin{array}{llll}7 & 1.5695 & 1.4989 & 0.0150\end{array}$

\begin{tabular}{l|lll}
5 & 1.9627 & 1.8964 & 0.0186
\end{tabular}

\begin{tabular}{l|lll}
3 & 2.1187 & 2.1469 & 0.0380
\end{tabular}
3.9493

3.1133

3.7597

4.4973

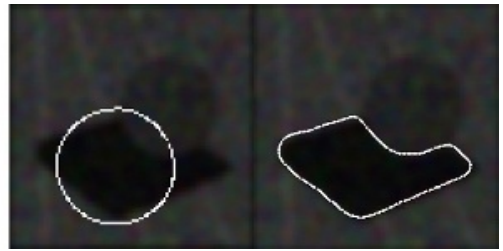

Mean Error Standard Min Error Max Error (dist/point) Deviation (dist/point) Deviation

$\begin{array}{llll}0.6301 & 0.6972 & 0.0009 & 1.7378\end{array}$

$\begin{array}{llll}1.0103 & 1.1175 & 0.0244 & 2.4629\end{array}$

$\begin{array}{llll}1.4057 & 1.4746 & 0.0020 & 3.0315\end{array}$

$\begin{array}{llll}2.1190 & 2.1552 & 0.0110 & 4.6364\end{array}$

$\begin{array}{llll}2.6271 & 2.5571 & 0.0976 & 3.7175\end{array}$

Fig. 5. CEPF segmentations on synthetic noisy images with known boundary for error analysis. Left: A bell shape with $\mathrm{SNR}=11 \mathrm{~dB}$. Right: A ball and plate with $\mathrm{SNR}=9 \mathrm{~dB}$.

However, the most obvious difference is that the Levelset representation only requires a scalar distance function $\Psi(\mathbf{x})$, while CEPF requires a vector value for every sample point. The gain from this extra memory/storage is that we have a greater flexibility on approximating the underlying continuous curve (Figures 3 and 4). Furthermore, increasing the size of the narrow band [4] of CEPF results in increased number of samples on the curve, while the number of curve elements is restricted by the sampling grid for Levelset. More importantly, the ultimate driving force for curve movement is limited to scalar data such as gradient magnitude in traditional Levelset methods, while CEPF is designed to be driven by vector form image information.

Discussion: Visually, the edges implied by opposite-pointing vectors are quite obvious towards human eyes looking from far apart, even from noisy GVF as shown in Figure 6. This is because strong edges will have greater diffusion towards their neighbors so that those affected neighbors appear to be more coherent. But simply applying a global threshold on the inner product between GVF vectors cannot detect all edge points because, if look closer on the GVF field, the angles sustained by opposite GVF vectors vary substantially which are due to the inconsistence of gradient magnitude along edge lines.

Experiments: Accuracy and robustness of the framework is assessed using synthetic images of various noises, as shown in Figure 5. Figure 6 shows the segmentation results of applying the CEPF framework to various medical images. Thresholding of edge strength is avoided by operating on an edge tendency field such as GVF. For images with stand-alone structure like a single mass in mammogram or endocardium in MRI, the CEPF works well on very low contrast images because the boundary edge strength is still dominating and resulting in proper GVF direction. But when irrelevant structure exists near target edges, GVF may be directed to other structures, depending on the relative edge strength and the amount of diffusion adopted in the GVF computing process. 

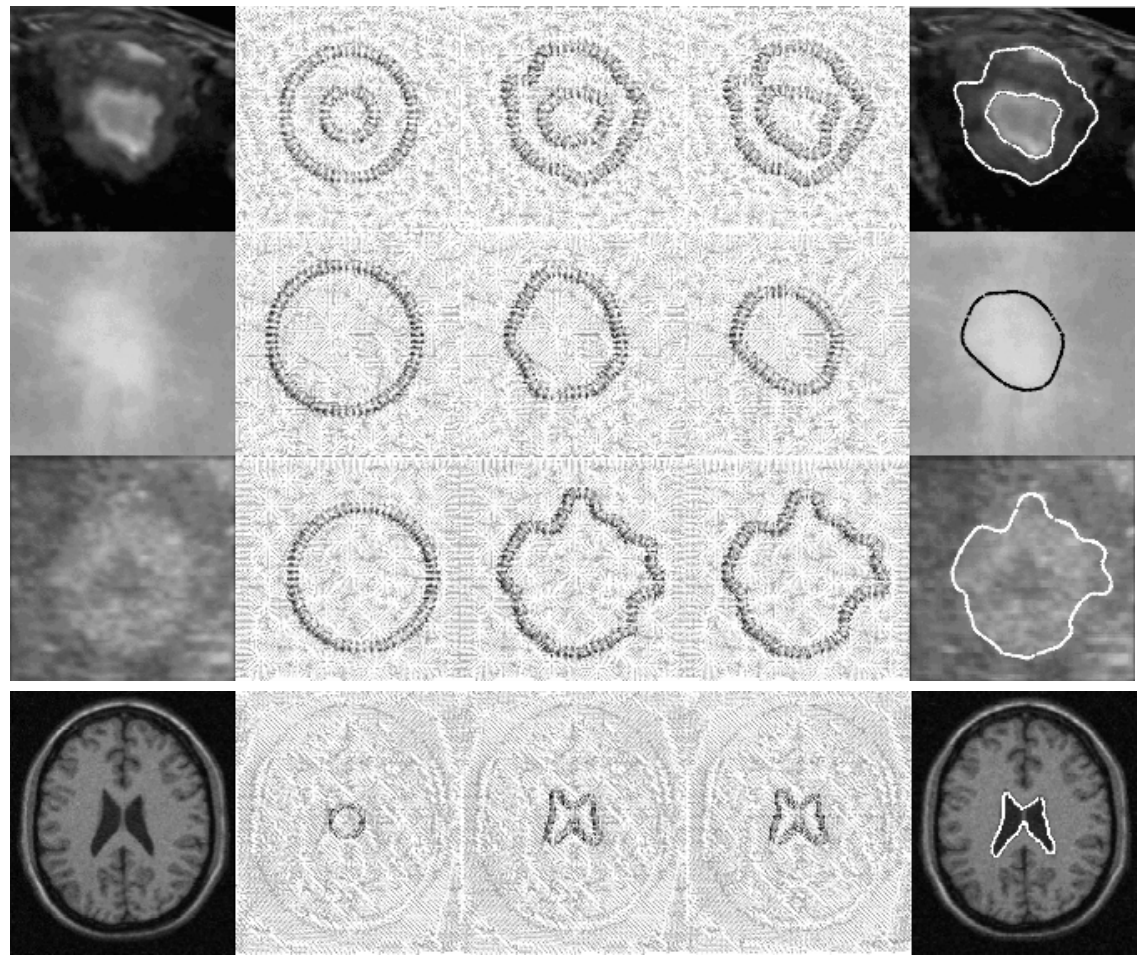

Fig. 6. CEPF segmentation results of various medical images. $1^{\text {st }}$ row: endocardium and epicardium from MRI image; $2^{\text {nd }}$ row: mass tumor from mammogram; $3^{\text {rd }}$ row: brain tumor from ultrasound image; and $4^{\text {th }}$ row: brain ventricle from synthetic MRI image of the BrainWeb [3] ( $T_{1}$ weighted, $1 \mathrm{~mm}$ slice thickness, and $9 \%$ noise).

If initial curve position fell into the influence domain of the irrelevant edges, the curve would stick to it and produce erroneous results. This can be improved by applying an additional biased field according to probability of correct edge occurrence.

Acknowledgement. This work is supported in part by Hong Kong Research Grant Council under Central Allocation Project CA00/01.EG01.

\section{References}

1. F. Chan and A. Vese. Active contours without edges. IEEE Transactions on Image Processing, 10(2):266-276, 2001.

2. M. Kass, A. Witkin, and D. Terzopoulos. Snakes: Active contour models. International Journal of Computer Vision, 1:321-331, 1987.

3. R.K.-S. Kwan, A.C. Evans, and G.B. Pike. MRI simulation-based evaluation of image-processing and classification methods. IEEE Transactions on Medical Imaging, 18(11):1085-1097, 1999. 
4. R. Malladi, J. A. Sethian, and B. C. Vemuri. Shape modeling with front porpagation: a level set approach. IEEE PAMI, 17(2):158-175, 1995.

5. Stanley Osher and Ronald Fedkiw. Level Set Methods and Dynamic Implicit Surfaces. Springer-Verlag, 2002.

6. N. Paragios, O. Mellina-Gottardo, and V. Ramesh. Gradient vector flow fast geodesic active contours. In IEEE ICCV'01, pages 67-73, 2001.

7. G. Ventura, J.X. Xu, and T. Belytschko. A vector level set method and new discontinuity approximations for crack growth by EFG. International Journal for Numerical Methods in Engineering, 54:923-944, 2002.

8. C. Xu and L. Prince. Snakes, shapes, and gradient vector flow. IEEE Transactions on Image Processing, 7(3):359-369, 1998. 